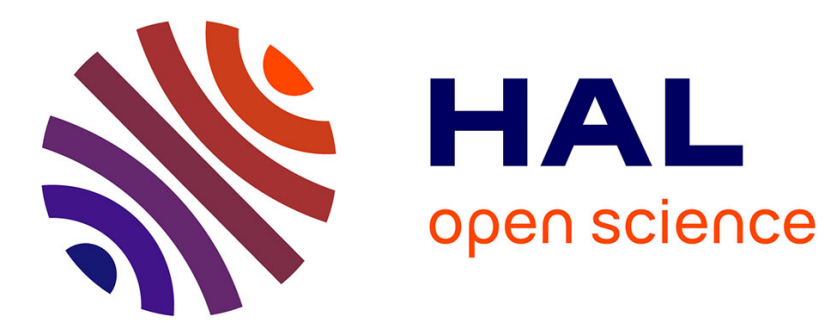

\title{
Mixing efficiency in stratified turbulence
}

\author{
Andrea Maffioli, Geert Brethouwer, Erik Lindborg
}

\section{To cite this version:}

Andrea Maffioli, Geert Brethouwer, Erik Lindborg. Mixing efficiency in stratified turbulence. Journal of Fluid Mechanics, 2016, 794, pp.R3. 10.1017/jfm.2016.206 . hal-03157651

\section{HAL Id: hal-03157651 \\ https://hal.science/hal-03157651}

Submitted on 10 Jun 2021

HAL is a multi-disciplinary open access archive for the deposit and dissemination of scientific research documents, whether they are published or not. The documents may come from teaching and research institutions in France or abroad, or from public or private research centers.
L'archive ouverte pluridisciplinaire HAL, est destinée au dépôt et à la diffusion de documents scientifiques de niveau recherche, publiés ou non, émanant des établissements d'enseignement et de recherche français ou étrangers, des laboratoires publics ou privés. 


\title{
Mixing efficiency in stratified turbulence
}

\author{
A. Maffioli $\dagger$, G. Brethouwer and E. Lindborg \\ Linné Flow Centre, Department of Mechanics, KTH, 10044 Stockholm, Sweden
}

(Received xx; revised xx; accepted xx)

We consider mixing of the density field in stratified turbulence and argue that, at sufficiently high Reynolds numbers, stationary turbulence will have mixing efficiency and closely related mixing coefficient described solely by the turbulent Froude number $F r=\epsilon_{k} /\left(N u^{2}\right)$, where $\epsilon_{k}$ is the kinetic energy dissipation, $u$ is a turbulent horizontal velocity scale and $N$ is the Brunt-Väisälä frequency. For $F r \gg 1$, in the limit of weakly stratified turbulence, we show through a simple scaling analysis that the mixing coefficient scales as $\Gamma \propto F r^{-2}$, where $\Gamma=\epsilon_{p} / \epsilon_{k}$ and $\epsilon_{p}$ is the potential energy dissipation. In the opposite limit of strongly stratified turbulence with $F r \ll 1$, we argue that $\Gamma$ should reach a constant value of order unity. We carry out direct numerical simulations of forced stratified turbulence across a range of $\mathrm{Fr}$ and confirm that at high $\mathrm{Fr}, \Gamma \propto \mathrm{Fr}^{-2}$, while at low $F r$ it approaches a constant value close to $\Gamma=0.33$. The parametrization of $\Gamma$ based on $R e_{b}$ due to Shih et al. (2005) can be re-interpreted in this light because the observed variation of $\Gamma$ in their study as well as in datasets from recent oceanic and atmospheric measurements occurs at a Froude number of order unity, close to the transition value $F r=0.3$ found in our simulations.

\section{Introduction}

The manifestation of mixing in the atmosphere and oceans takes different forms from phyto-plancton blooms in the oceans to the creation of well-mixed fronts in the atmosphere. Mixing in the atmosphere and oceans is a key factor to consider when estimating global energetics. Central to the attempt of quantifying mixing in the oceans is the concept of eddy diffusivity, defined as $K_{\rho}=B / N^{2}$ (Osborn \& Cox 1972) where $B=-\left\langle b u_{z}\right\rangle$ is the buoyancy flux, $b=-\rho^{\prime} g / \rho_{0}$ being the buoyancy acceleration related to the density perturbation $\rho^{\prime}$ from the background stratification and $u_{z}$ being the vertical velocity perturbation. The idea is that the buoyancy flux can be modelled as $B=$ $-\left\langle b u_{z}\right\rangle=K_{\rho} d \bar{b} / d z$, where the mean buoyancy gradient is $d \bar{b} / d z=N^{2}$. Osborn $(1980)$ inspected the turbulent kinetic energy equation in the presence of buoyancy and assuming steady-state conditions introduced the flux Richardson number $R i_{f}=B /\left(B+\epsilon_{k}\right)$, which is the ratio of buoyancy flux to turbulence production by the mean flow and can be thought of as a mixing efficiency. A related quantity is the mixing coefficient $\Gamma=B / \epsilon_{k}$, so that $K_{\rho}=\Gamma \epsilon_{k} / N^{2}$. A constant mixing efficiency $\eta=R i_{f}=0.17$ was assumed by Osborn (1980) leading to a mixing coefficient $\Gamma=R i_{f} /\left(1-R i_{f}\right)=0.2$, a value which has been widely used in oceanographic applications ever since. Salehipour \& Peltier (2015) suggested the use of $\epsilon_{p}$ instead of the buoyancy flux $B$ when calculating the mixing coefficient, because the buoyancy flux contains both reversible and irreversible exchanges of kinetic and available potential energy, while we are interested in the irreversible conversion of available potential energy into background potential energy, quantified by $\epsilon_{p}$. Throughout the paper we therefore use the definitions $\Gamma=\epsilon_{p} / \epsilon_{k}$ for the mixing coefficient and $\eta=\epsilon_{p} /\left(\epsilon_{k}+\epsilon_{p}\right)$ for the mixing efficiency. Direct support for an eddy

$\dagger$ Email address for correspondence: maffioli@mech.kth.se 
diffusivity of the form $K_{\rho}=\epsilon_{p} / N^{2}=\Gamma \epsilon_{k} / N^{2}$ was provided by the work of Lindborg $\&$ Brethouwer (2008) who derive an analytical expression for the mean square particle displacement $1 / 2\left\langle\delta z^{2}\right\rangle$, which increases linearly in time, the constant of proportionality being $K_{\rho}$.

The parameters that could conceivably affect mixing in stratified turbulence are the buoyancy Reynolds number $R e_{b}$, the turbulent Froude number $F r$ and the Reynolds number $R e$. Taking advantage of the estimate $\epsilon_{k} \sim u^{3} / \ell$, we define these as

$$
R e_{b}=\frac{\epsilon_{k}}{\nu N^{2}}, \quad F r=\frac{\epsilon_{k}}{N u^{2}}, \quad R e=\frac{u^{4}}{\nu \epsilon_{k}},
$$

These definitions differentiate themselves from the more classical definitions using a turbulent lengthscale $\ell$. Throughout the paper $u$ and $\ell$ are considered to be horizontal scales so that the results generalize to the case of strongly stratified turbulence and its anisotropic conditions. In this case then $F r$ represents a horizontal Froude number, as opposed to the vertical Froude number $F r_{v}=u /\left(N \ell_{v}\right)$ based on a vertical lengthscale $\ell_{v}$. As shown by Billant \& Chomaz (2001), $F r_{v}=\mathcal{O}(1)$ in the limit of low $F r$ and therefore $F r_{v}$ does not further influence the dynamics. As pointed out for example by Ivey et al. (2008) the three parameters listed in equation (1.1) are not independent since $R e_{b}=R e F^{2}$. In problems of mixing through a density discontinuity a relevant parameter is the bulk Richardson number $R i_{b}=g \Delta \rho \ell /\left(\rho_{0} u^{2}\right)$ where $\Delta \rho$ is the density jump across the interface and $\rho_{0}$ is a reference density. If we substitute $\Delta \rho / \ell$ with the continuous stratification density gradient $|d \bar{\rho} / d z|$ it is clear that $R i_{b} \sim N^{2} \ell^{2} / u^{2} \sim F r^{-2}$ using the definition of $N=\sqrt{-\left(g / \rho_{0}\right) d \bar{\rho} / d z}$. Hence problems with a density interface can also effectively be characterized by the parameters given in (1.1).

Since the seminal work of Osborn \& Cox (1972); Osborn (1980) it has been found that mixing efficiency and mixing coefficient are not constants but vary in a certain parameter range. Barry et al. (2001) found a variation of the mixing coefficient in their grid stirring experiments for buoyancy Reynolds numbers $R e_{b}=\epsilon_{k} /\left(\nu N^{2}\right)>300$ that was well described by $\Gamma \propto R e_{b}^{-2 / 3}$. In their DNS of stratified shear flow, Shih et al. (2005) found a constant mixing coefficient $\Gamma \approx 0.2$ for $7<R e_{b}<100$ but a varying mixing coefficient going as $\Gamma \propto R e_{b}^{-1 / 2}$ for $R e_{b}>100$. Ocean field measurements by Davis \& Moninsmith (2011); Walter et al. (2014) have found similar variations of $\Gamma \propto \operatorname{Re}_{b}^{-1 / 2}$ at high $R e_{b}>100$. Atmospheric boundary layer measurements within the vertical transport and mixing experiment (VTMX) also have a similar variation of $\Gamma$ with $R e_{b}$ but now at $R e_{b}>10^{4}$, suggesting a completely different bound on the buoyancy Reynolds number (see Lozovatsky \& Fernando 2013). Recently, Mater \& Venayagamoorthy (2014) have suggested that a multi-parameter approach is more suitable to describe mixing processes in stratified turbulence.

Classical parametrizations of mixing have focused on the bulk Richardson number $R i_{b}$, which is closely related to the turbulent Froude number since $R i_{b} \sim F r^{-2}$. In experiments of mixing across a density interface by Turner (1968); Kato \& Philipps (1969) the focus was on the entrainment velocity $u_{e}$ across the interface. This is closely related to the mixing efficiency and it can be shown that $\eta=R i_{b}\left(u_{e} / u\right) \sim F r^{-2}\left(u_{e} / u\right.$ ) (see Turner 1973). In the limit of weak stratification, corresponding to high values of Fr, Turner (1968) found that the normalized entrainment velocity $u_{e} / u$ reaches a constant, implying the result $\eta \propto \mathrm{Fr}^{-2}$ for weakly stratified turbulence. This result has also been suggested by Holford \& Linden (1999) who state that the buoyancy field behaves as a passive scalar in the limit of high $F r$. At the other end of the spectrum, strong stratification and low $F r$ lead to an entrainment velocity $u_{e} / u \propto R i_{b}^{-1} \sim F r^{2}$ in the experiments by 
Kato \& Philipps (1969). This means a constant mixing efficiency $\eta$ in the limit of strongly stratified turbulence, which has been confirmed by several more recent experiments (Park et al. 1994; Oglethorpe et al. 2013; Olsthoorn \& Dalziel 2015).

Hence two parametrizations of mixing exist: a more classical one based on $R i_{b}$ and therefore essentially on the turbulent Froude number $\mathrm{Fr}$ and a more recent one based on $R e_{b}$. We now turn to the evidence from scaling of the equations of motion to try and shed light on this debate.

The Boussinesq set of equations for a linearly stratified fluid is given by:

$$
\begin{aligned}
\frac{\partial \mathbf{u}}{\partial t}+\mathbf{u} \cdot \nabla \mathbf{u} & =-\frac{1}{\rho_{0}} \nabla p+b \mathbf{e}_{z}+\nu \nabla^{2} \mathbf{u}, \\
\frac{\partial b}{\partial t}+\mathbf{u} \cdot \nabla b & =-N^{2} u_{z}+\mathcal{D} \nabla^{2} b .
\end{aligned}
$$

We consider first the limit of weak stratification and high $F r$ where horizontal and vertical lengthscales can be assumed to be approximately equal as can be done for horizontal and vertical velocity scales. From the buoyancy equation (1.3) a simple balance between advection $\mathbf{u} \cdot \nabla b$ and the background stratification term $N^{2} u_{z}$ leads to a scaling for the buoyancy as $b \sim N^{2} \ell$. Comparing this to the advection term in the Boussinesq momentum equation (1.2) $\mathbf{u} \cdot \nabla \mathbf{u} \sim u^{2} / \ell$ it is clear that their ratio is $\left(N^{2} \ell\right) /\left(u^{2} / \ell\right)=N^{2} \ell^{2} / u^{2} \sim$ $\mathrm{Fr}^{-2}$. Hence in the limit of $\mathrm{Fr} \gg 1$, the buoyancy term can be neglected to leading order in the momentum equation and buoyancy effectively behaves as a passive scalar in the presence of a mean scalar gradient $N^{2}$ as pointed out by Holford \& Linden (1999). It is possible from equations (1.2)-(1.3) to form equations for the turbulent kinetic energy and potential energy. At high Reynolds number and high Peclet number, the kinetic and potential energy dissipation rates reach a finite and positive limit that is of the same order as the advection term in these energy equations:

$$
\begin{aligned}
& \epsilon_{k} \sim \mathbf{u} \cdot \nabla\left(\frac{|\mathbf{u}|^{2}}{2}\right) \sim \frac{u^{3}}{\ell}, \\
& \epsilon_{p} \sim \mathbf{u} \cdot \nabla\left(\frac{b^{2}}{2 N^{2}}\right) \sim \frac{b^{2} u}{N^{2} \ell} \sim N^{2} u \ell .
\end{aligned}
$$

From these scalings it follows that the mixing coefficient $\Gamma=\epsilon_{p} / \epsilon_{k} \sim\left(N^{2} u \ell\right) /\left(u^{3} / \ell\right)=$ $N^{2} \ell^{2} / u^{2} \sim F^{-2}$ in the limit of high $F r$. This result is analogous to that obtained when considering Turner's experiment. The Prandtl number $\operatorname{Pr}=\nu / \mathcal{D}$ does not affect the analysis, because we assume that the Reynolds number $R e$ and Peclet number $P e=$ $\operatorname{Re} \operatorname{Pr}$ are both sufficiently high that the kinetic and potential energy dissipation rates become independent of viscosity $\nu$ and diffusivity $\mathcal{D}$, respectively.

The strongly stratified turbulence theory was developed by several researchers in this field; its two main conditions are $F r \ll 1$ and $R e_{b} \gg 1$ and these conditions both have to be met within the theoretical framework (developed by Billant \& Chomaz 2001; Lindborg 2006). The scaling analyses behind this work have proven to be able to describe the layered large-scale appearance of strongly stratified turbulence, observed previously by many authors and reviewed extensively by Riley \& Lelong (2000). A consensus has emerged recently that for values $R e_{b}>10$ stratified turbulence has the expected form of the horizontal energy spectrum $E_{h}\left(k_{h}\right) \sim \epsilon_{k}^{2 / 3} k_{h}^{-5 / 3}$ and that the vertical lengthscale is approximately equal to $\ell_{v}=u / N$. This has been shown in many numerical works in the last ten years (Brethouwer et al. 2007; Waite 2011; Bartello \& Tobias 2013; Augier et al. 2015; Maffioli \& Davidson 2015). Brethouwer et al. (2007) tested many of the 
predicted results of the strongly stratified turbulence theory and plotted, for example, the anisotropy of the dissipation $S_{\text {diss }}$ against $R e_{b}$ in their forced stratified DNS simulations. This quantity is defined as $S_{\text {diss }}=\nu\left\langle\left(\partial u_{x} / \partial z\right)^{2}+\left(\partial u_{y} / \partial z\right)^{2}\right\rangle / \epsilon_{k}$ and represents the ratio of dissipation due to vertical gradients to the overall dissipation. The authors of this work found that $S_{\text {diss }}$ rapidly goes towards the isotropic value of $4 / 15 \approx 0.267$ (Taylor $1935)$ as the buoyancy Reynolds number is increased. Similar results were obtained in decaying stratified turbulence by Riley \& de Bruyn Kops (2003); Maffioli \& Davidson (2015) who both found values close to $S_{\text {diss }} \approx 0.41$ at times when the stratified turbulence in their DNS was most vigorous and $R e_{b}=5-9$. Furthermore, as discussed extensively in Smyth \& Moum (2000); Smyth et al. (2005), in the case of stratified mixing layers a good condition for determining if there is active three-dimensional turbulence at the small scales is that $R e_{b}>20$. The issue of whether the isotropic limit of the dissipation terms will be reached in strongly stratified turbulence is still being studied and constitutes an active area of research. Note however that the isotropic limit is not necessary for our main scaling analysis to be applicable because the scaling is based on the classical assumption that the dissipation rates reach a finite and positive limit at high Reynolds number, irrespective of the values of viscosity and diffusivity.

We now proceed to the main part of this paper that is concerned with estimating the dependency of $\Gamma$ on the important physical parameters of the problem. Considering our novel scaling analysis at high $F r$ and the above arguments, it is our contention that in stratified turbulence the mixing efficiency is described by the Froude number only. The buoyancy Reynolds number $R e_{b}$ should not play any role. To test this theoretical reasoning, we have performed DNS of stratified turbulence across a large range of Froude numbers and Reynolds numbers.

\section{Numerical methodology}

We perform direct numerical simulations (DNS) of turbulence in a linearly stratified fluid, with constant Brunt-Väisälä frequency $N$. The equations which are solved directly are the Boussinesq set of equations (1.2)-(1.3) together with the continuity equation $\nabla \cdot \mathbf{u}=0$. We have included a body force $\mathbf{f}$ in the Boussinesq momentum equation (1.2) to ensure that the turbulence reaches statistical stationarity. These equations are solved using a pseudo-spectral method based on Rogallo's algorithm (Rogallo 1981). Time advancement is carried out using a second-order Runge-Kutta predictor-corrector integration scheme, while the viscous and diffusive terms are integrated exactly by using suitable integrating factors. De-aliasing of the non-linear terms is performed using a combination of truncation and phase-shifting (for more details see Rogallo 1981).

The turbulence is kept in a steady state through the use of forcing. We use random forcing that is uncorrelated in time, in a way that the physical location of the body force keeps changing in time. Most of the simulations are at high $\mathrm{Fr}$, for which we use isotropic forcing in spherical shells of radius $k_{f}^{\max }=5$. The forcing is mostly concentrated at smaller wavenumbers with a forcing power spectrum $P(k)=\alpha^{2} \exp \left(-\left(k-k_{f}^{\text {peak }}\right)^{2}\right)$ with $k_{f}^{\text {peak }}=3$. The value of $\alpha$ is determined at every timestep to ensure that the forcing power $P_{f}=\sum_{\text {all } \mathbf{k}}\left(\hat{\mathbf{u}}^{*} \cdot \hat{\mathbf{f}}+1 / 2|\hat{\mathbf{f}}|^{2} \Delta t\right)$ is kept to a constant value. Since $\hat{\mathbf{f}} \propto \alpha$ this results in a quadratic equation for $\alpha$, which we solve at every timestep. Of the two roots for $\alpha$ we choose the one with minimum absolute value, which therefore minimizes the magnitude of the force; we find this technique reduces the oscillations in time for most quantities of interest, such as $\epsilon_{k}, \epsilon_{p}$ and RMS values of the velocity components. At low Froude 


\begin{tabular}{lccccccc}
\hline & & & & & & \\
Run & $N_{x}=N_{y}$ & $N_{z}$ & $F r$ & $R e_{b}$ & $R e_{\lambda}$ & $R e$ & forcing \\
R1kF2.9 & 96 & 96 & 2.90 & 1010 & 42 & 120 & iso \\
R1kF1.6 & 192 & 192 & 1.64 & 990 & 74 & 370 & iso \\
R1kF0.9 & 384 & 384 & 0.94 & 980 & 129 & 1120 & iso \\
R1kF0.5 & 768 & 768 & 0.52 & 960 & 229 & 3480 & iso \\
R1kF0.3 & 1536 & 1536 & 0.29 & 990 & 423 & 11930 & iso \\
1024F0.7 & 1024 & 1024 & 0.70 & 2340 & 266 & 4730 & iso \\
1024F1.6 & 1024 & 1024 & 1.58 & 10430 & 250 & 4180 & iso \\
1024F3.1 & 1024 & 1024 & 3.10 & 37370 & 242 & 3900 & iso \\
1024F5.9 & 1024 & 1024 & 5.86 & 133430 & 241 & 3880 & iso \\
1024F12 & 1024 & 1024 & 11.97 & 537250 & 237 & 3750 & iso \\
256F2.9 & 256 & 256 & 2.85 & 4190 & 88 & 520 & iso \\
512F3 & 512 & 512 & 3.03 & 13370 & 148 & 1460 & iso \\
R200F0.14 & 1024 & 1024 & 0.141 & 200 & 390 & 10130 & vort \\
R57F0.09 & 1024 & 1024 & 0.091 & 57 & 319 & 6760 & vort \\
R14F0.04 & 1024 & 512 & 0.044 & 14 & 324 & 7000 & vort \\
R15F0.03 & 2048 & 512 & 0.035 & 15 & 432 & 12420 & vort \\
R17F0.02 & 4096 & 1024 & 0.020 & 17 & 805 & 43180 & vort
\end{tabular}

TABLE 1. List of DNS runs performed: relevant non-dimensional parameters and type of forcing.

numbers $F r<0.2$, we found that isotropic forcing leads to non-stationary solutions, with growth of energy in the shear modes (modes with $k_{x}=k_{y}=0$ ) that quickly dominate the overall kinetic energy. We therefore utilize vortical forcing concentrated in the vertically rotational modes with $k_{z}=0$. This type of forcing in modes with $k_{z}=0$ and finite and small $k_{h}$ takes a long time to leak energy to the shear modes, with finite and small $k_{z}$ and $k_{h}=0$, and hence a quasi-stationary state can be reached at intermediate times. Vortical forcing has been used by numerous authors when studying stratified turbulence at low Fr (see Waite \& Bartello 2004; Brethouwer et al. 2007; Augier et al. 2015).

Most simulations are run on cubic domains except for the three simulations with strongest stratification for which we take advantage of the anisotropy of the lengthscales and use rectangular domains with $L_{z}<L_{h}$. In all cases, since we expect isotropic dissipation at high $R e$, the grid spacing is constant in all directions and it is chosen to ensure $k_{\max } \eta=1.5$. The Prandtl number in all cases is $\operatorname{Pr}=1$. The list of all simulations that have been performed for this study is given in table 1 together with the important physical parameters for each run. The strongly stratified runs are performed on increasingly large grids in order to meet the condition $R e_{b}>10$ as $F r$ is decreased. As a result our most strongly stratified run with $F r=0.02$ and $R e_{b}=17$ requires a grid of $4096^{2} \times 1024$ collocation points. The values quoted are time averages over the stationary period of each simulation as are all the values plotted in the next section, in which we discuss the results of the various stratified DNS performed.

\section{Results of DNS runs}

\subsection{Variation of mixing coefficient at constant $R_{b}$}

We have performed simulations at constant buoyancy Reynolds number and at different Froude numbers. This was achieved by using different number of grid points for each simulation and hence varying also the Reynolds number while keeping $R e_{b} \approx 1000$. This value of $R e_{b}$ is well within the energetic-regime of Barry et al. (2001); Shih et al. (2005) so that the mixing coefficient is expected to not be constant. As shown in figure 1, we 


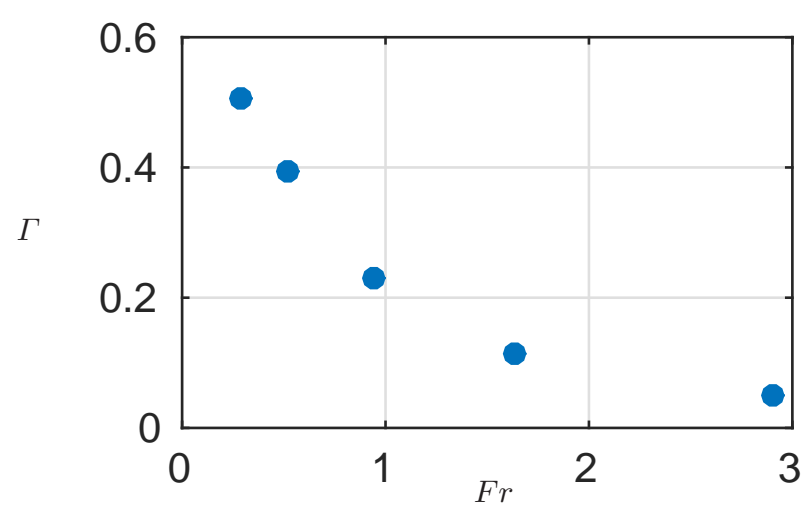

Figure 1. Mixing coefficient as a function of Froude number at a constant $R e_{b} \approx 1000$. Each point corresponds to one of the five simulations labelled R1kF2.9 through to R1kF0.3.

indeed find a variation of $\Gamma$ across the five runs but this can now not be due to variations in $R e_{b}$, which is kept constant, but rather is due to a changing $F r$. The values of $\Gamma$ span an order of magnitude as do the values of $F r$ in the simulations. At $F r=0.29$ we have $\Gamma=0.51$, a high value compared to the often quoted $\Gamma=0.2$ value (Osborn 1980). At the other end of the $F r$-range, $F r=2.9$ (but at the same $R e_{b}$ ), we have $\Gamma=0.05$. Such a low value of the mixing coefficient is a result of the weak stratification meaning that there is not much of a density difference for the turbulent flow to mix.

\subsection{Mixing coefficient at varying Reynolds numbers}

The kinetic and potential energy dissipation rates reach the finite and positive limits given in equations (1.4)-(1.5) at high- $R e_{\lambda}$, the Reynolds number based on the Taylor microscale, defined as $R e_{\lambda}=u \lambda / \nu$ with $\lambda=\left(u^{2} /<\left(\partial u_{x} / \partial x\right)^{2}>\right)^{1 / 2}$. This was shown by Donzis et al. (2005) for turbulence with a passive scalar and is expected to be true also for stratified turbulence. If $R e_{\lambda}$ is not high enough both the non-dimensional dissipations become a function of the Reynolds number, that is $\epsilon_{k} \ell / u^{3}=f\left(R e_{\lambda}\right)$ and $\epsilon_{p} \ell /\left(u b^{2} / N^{2}\right)=$ $g\left(R e_{\lambda}\right)$, and these functions are given in Donzis et al. (2005) in the case of passive scalar advection. Because the result $\Gamma \propto F r^{-2}$ follows from the dissipation scalings in equations (1.4)-(1.5), at finite Reynolds number we expect instead $\Gamma=h\left(F r, \operatorname{Re}_{\lambda}\right)$ and the mixing efficiency now depends not only on the Froude number but also on the Taylor scale Reynolds number.

To confirm these ideas we consider first the non-dimensionalized dissipation rates given in figure 2 for four runs at constant $F r \approx 3$ but performed on successively larger grids so as to increase $R e_{\lambda}$ from low values to $R e_{\lambda} \approx 240$. This value is considered to be a good high value above which both dissipations reach a constant limit when nondimensionalized (see Donzis et al. 2005; de Bruyn Kops 2015). In making the dissipations non-dimensional we use the standard isotropic definition of the turbulent lengthscale, $\ell=\left(\pi / u^{2}\right) \int(E(k) / k) d k$ based on the 3-D energy spectrum $E(k)$, expected to be valid at this high $\mathrm{Fr}$. We find a non-dimensionalized kinetic energy dissipation that is remarkably close to the form found by Donzis et al. (2005) in their collection of DNS (the exact same constants as in their paper are used in the curve shown in figure 2(a)). Also the nondimensionalized potential energy dissipation is similar to the prediction of Donzis et al. (2005) for the non-dimensionalized dissipation of passive scalar variance, confirming that buoyancy behaves as a passive scalar at high Froude numbers. 


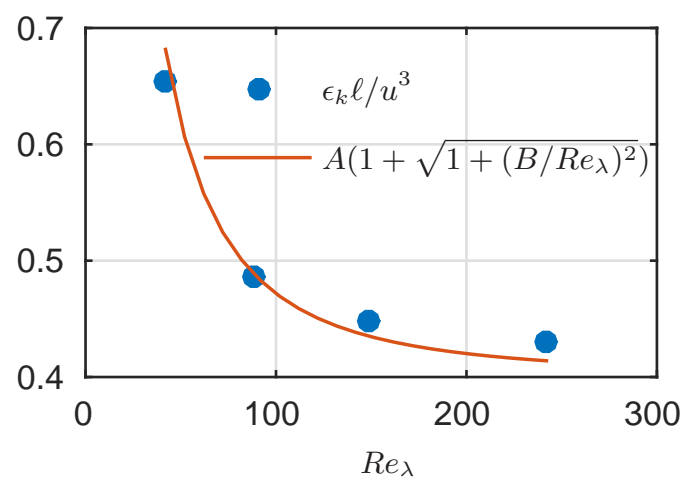

(a)

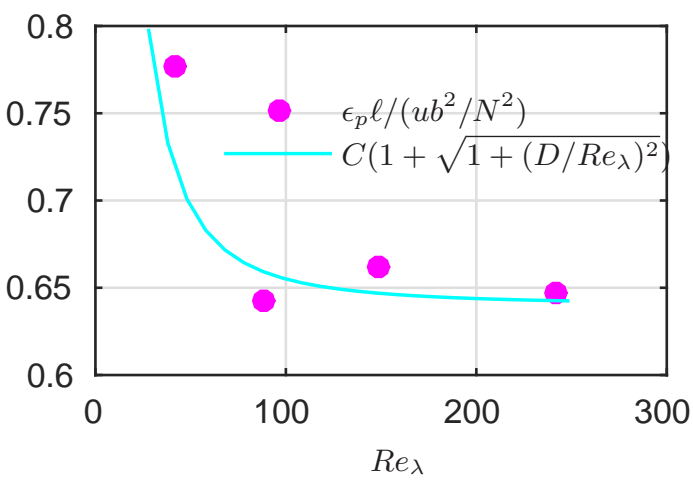

(b)

FIgURE 2. (a) Kinetic energy dissipation and (b) potential energy dissipation, both non-dimensionalized, for 4 runs with $F r \approx 3$. The relevant DNS runs are R1kF2.9, 256F2.9, 512F3 and 1024F3.1. The curves represent the fit proposed by Donzis et al. (2005) with the same constants in the case of $\epsilon_{k} \ell / u^{3}$, and the same value for $D$ but a slightly different value for $C$ in the case of $\epsilon_{p} \ell /\left(u b^{2} / N^{2}\right)$ (we use $C=0.31$ ).

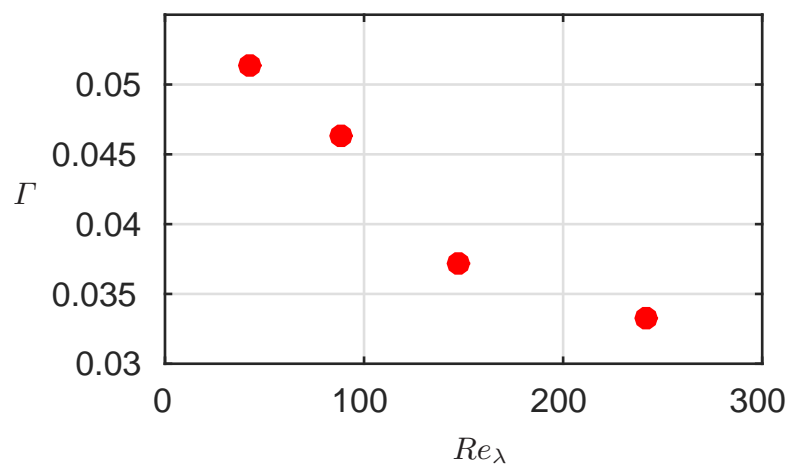

Figure 3. Mixing coefficient $\Gamma$ as a function of $R e_{\lambda}$ at a constant $F r \approx 3$.

The next step is to check that, for these four simulations at small to moderate $R e_{\lambda}$, also the mixing coefficient $\Gamma$ changes, approaching a constant at the highest $R e_{\lambda}$. The steady-state value of $\Gamma$ obtained for the four runs is given in figure 3, and there is clearly a significant variation, with $\Gamma$ decreasing for increasing values of $R e_{\lambda}$ and, possibly, the approach of a constant value $\Gamma \approx 0.033$ at the highest $R e_{\lambda}=242$.

\subsection{Mixing coefficient at high $R e_{\lambda}$}

We now consider only the runs with $R e_{\lambda}>200$, for which the dissipation rates $\epsilon_{k}$ and $\epsilon_{p}$ have approximately reached their respective finite and positive limits.

In figure 4 we show $\Gamma$ as a function of $F r$ for these runs. If we focus on the high- $F r$ behaviour we see that indeed $\Gamma \propto F r^{-2}$ for $F r>1$, which confirms the scaling arguments delineated in $\S 1$. These scaling arguments have as a first result $E_{P} / E_{K} \sim E_{P} / u^{2} \propto F r^{-2}$ and this is also confirmed by the simulations for $F r>1$ as shown in figure 5 . Of course our simulations are in a stationary state, which makes them easier to analyse, while time-evolving simulations such as those of Shih et al. (2005); Salehipour \& Peltier (2015) have an extra degree of freedom. It may be that some definition of the Reynolds number may be important to describe the decay of the turbulence, or the "age of a mixing event" 


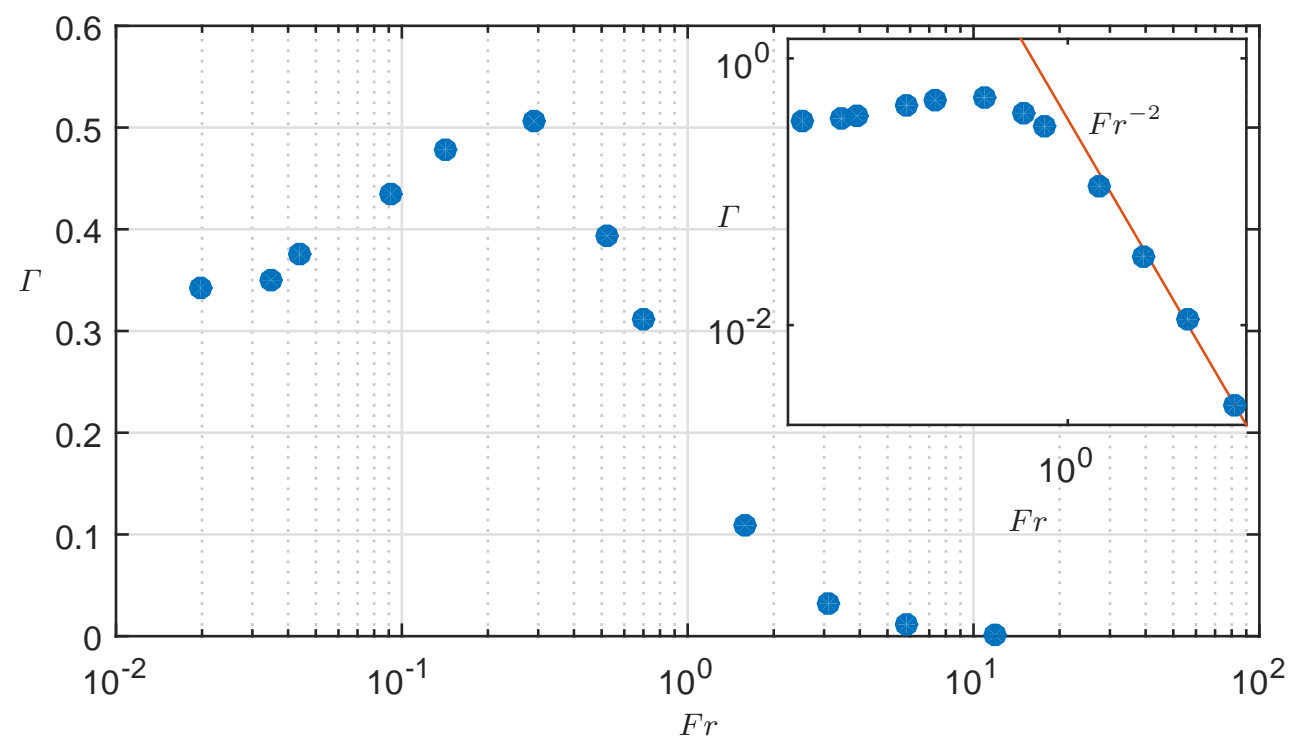

Figure 4. Mixing coefficient as a function of Froude number (log-log plot shown in inset).

as described by Smyth \& Moum (2000), in this case. However, at $F r>1$ the relevant Reynolds number should not be the buoyancy Reynolds number. This follows from a simple argument, the Ozmidov scale $\ell_{\mathrm{oz}} \sim F r^{3 / 2} \ell>\ell \approx L_{\text {box }}$ for $F r>1$. The buoyancy Reynolds number is a measure of the dynamic range from the Kolmogorov scale through to $\ell_{\mathrm{oz}}$ since it can be written as $R e_{b}=\left(\ell_{\mathrm{oz}} / \eta\right)^{4 / 3}$. However, the Ozmidov scale is now larger than $\ell$, the largest physical scale in the problem, and for this reason $R e_{b}$ does not have a physical influence on the stratified turbulence being simulated.

In the limit of low $F r$ and strong stratification, we expect the mixing coefficient to reach a constant value as in the experiments by Park et al. (1994); Oglethorpe et al. (2013); Olsthoorn \& Dalziel (2015) as long as $R e_{b}>10$. We have chosen this criterion because there is evidence that for $R e_{b}=\mathcal{O}(10)$ the turbulence starts showing the characteristics of strongly-stratified turbulence as discussed in $\S 1$. In figure 4 we see that $\Gamma$ has a maximum around $F r=0.3$ and then drops as the stratification is increased. Our results confirm that $\Gamma$ indeed approaches a constant value $\Gamma \approx 0.33$ as $F r \rightarrow 0$. The ratio $E_{P} / u^{2}$ is also predicted to be a constant of order unity by the strongly stratified turbulence theory (see Billant \& Chomaz 2001). From figure 5 this ratio appears to slowly go to a constant value $E_{P} / u^{2} \approx 0.15$ for $F r<0.3$.

\section{Discussion and conclusions}

There is a revealing relationship linking $F r$ and $R e_{b}$ in the limit of high Reynolds number when the dissipative scales are isotropic and $\epsilon_{k}=15 \nu<\left(\partial u_{x} / \partial x\right)^{2}>$ :

$$
F r=\frac{\sqrt{15 R e_{b}}}{R e_{\lambda}}
$$

In the case of the DNS of Shih et al. (2005) $R e_{\lambda} \approx 90$ for most of their simulations. Considering their value of $R e_{b}=100$ at which $\Gamma$ starts to decrease, we can use 


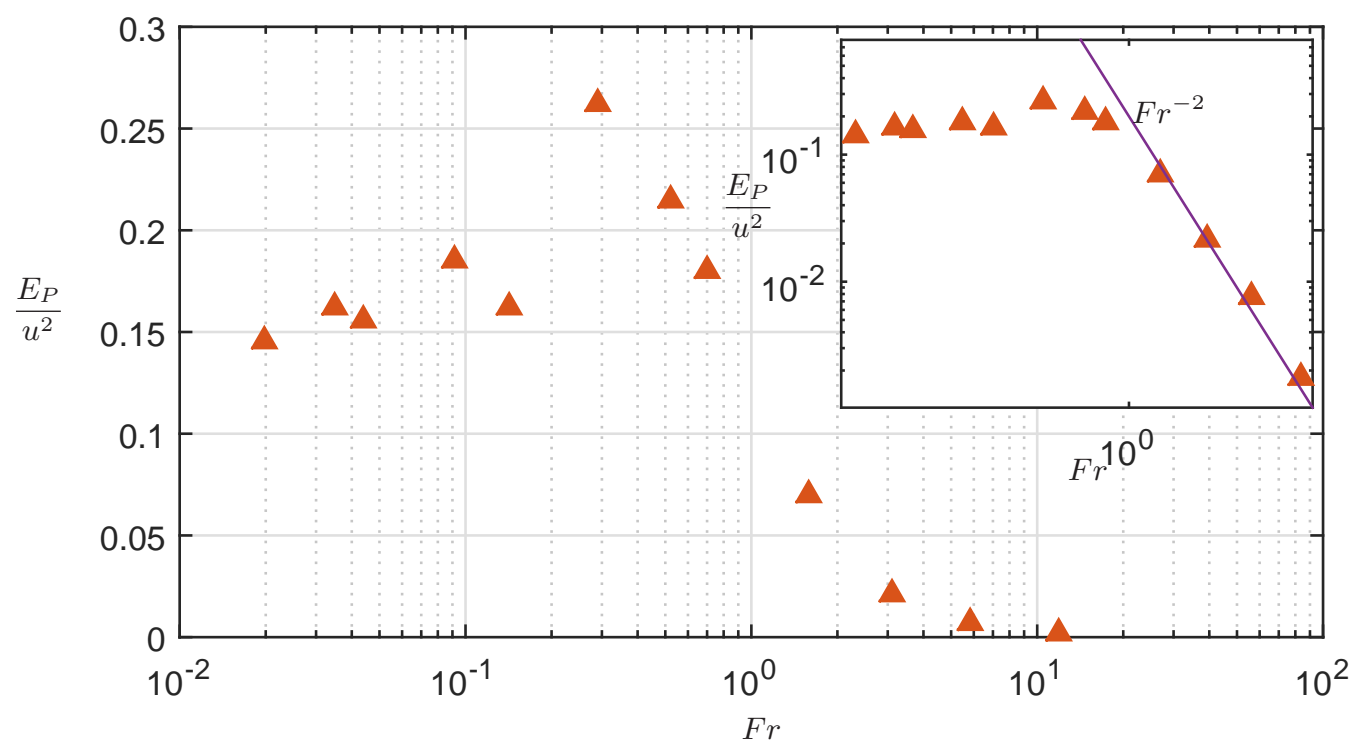

FiguRE 5. Ratio of potential energy to horizontal kinetic energy as a function of Froude number (log-log plot shown in inset).

equation (4.1) to find that the corresponding turbulent Froude number is $F r=0.45$, which is close to the value of $F r=0.3$ at which we observed the drop in $\Gamma$ (see figure 4 ).

We can take this a step further and try to estimate the value of $F r$ at which $\Gamma$ starts varying in ocean and atmosphere field data. Davis \& Moninsmith (2011) report turbulence Reynolds numbers greater than $R e_{\ell}=1500$ in their ocean measurements. Using $R e_{\ell}=u \ell / \nu \sim u^{4} /\left(\nu \epsilon_{k}\right)=15 R e_{\lambda}^{2}$ we can make the estimate $R e_{\lambda} \approx \sqrt{15 R e}=150$, not much larger than the value from the DNS of Shih et al. (2005). The variation in $\Gamma$ was observed for $R e_{b}>100$ or for $F r>\sqrt{15 R e_{b}} / R e_{\lambda}=0.26$, again close to our transition Froude number.

We finally consider the atmospheric data of Lozovatsky \& Fernando (2013). From the data of the VTMX experiment in Monti et al. (2002), night-time values for the horizontal turbulent velocity were $u \approx 0.3 \mathrm{~m} / \mathrm{s}$, while a value for the dissipation can be inferred from the compensated spectra in Figure 4(b) of Lozovatsky \& Fernando (2013) as $\epsilon_{k} \approx 1.8 \times 10^{-3} \mathrm{~m}^{2} / \mathrm{s}^{3}$. Using $\nu=1.4 \times 10^{-5} \mathrm{~m}^{2} / \mathrm{s}$ for air at $10^{\circ} \mathrm{C}$ one obtains a value for the Taylor microscale as $\lambda=0.1 \mathrm{~m}$ and $R e_{\lambda} \approx 2000$. This finally gives a transition Froude number as $F r=0.39$ corresponding to $R e_{b}=40,000$. So for completely different transition values based on buoyancy Reynolds number (to which we can add $R e_{b}=1000$ from the present DNS) we have similar value of turbulent Froude number close to $\mathrm{Fr} \approx 0.3$ at which the mixing coefficient starts dropping considerably. The variation in $\Gamma$ does therefore appear to be a Froude number effect. It remains to clearly show $\Gamma \propto \mathrm{Fr}^{-2}$ in field data, which is essential in our eyes to finish resolving this open issue in stratified turbulent mixing. The first evidence in this direction comes from the work of Wells et al. (2010) who find $\Gamma \sim F r^{-2}$ at high $F r$ in the case of oceanic gravity currents.

In conclusion, we have presented results from direct numerical simulations of constant$N$ forced stratified turbulence covering almost 3 orders of magnitude in $\mathrm{Fr}$ and a vast range of $R e_{b}$. The simulations at high $R e_{\lambda}$ show a clear behaviour of the mixing coefficient 
as $\Gamma \propto \mathrm{Fr}^{-2}$ for $\mathrm{Fr}>1$, confirming the scaling analysis for weakly stratified turbulence. In the opposite limit, $F r<1$ a peak in $\Gamma$ is found at $F r \approx 0.3$ with a high value of mixing coefficient $\Gamma=0.51$, significantly larger than the $\Gamma=0.2$ value that is commonly used in oceanographic applications. The mixing coefficient then drops to values around $\Gamma=0.33$; these are still high values and are due to the presence of a strong stratification, which high- $R e_{b}$ turbulence is able to mix efficiently. To what extent our results can be generalized to all types of flows is an open question. We find it very likely that the general dependence of $\Gamma$ on $F r$ in the two limits $F r \ll 1$ and $F r \gg 1$ will show some degree of universality.

We would like to thank P.K. Yeung for providing us with the DNS code used for this study. We thank Pierre Augier for helpful discussions on mixing efficiency. We are indebted to the Leverhulme Trust network on Waves \& Turbulence headed by Peter Davidson for the many discussions it generated between its participants. We acknowledge PRACE for awarding us via the STRATFORCE project access to the Fermi cluster at CINECA in Italy. Computational resources at PDC in Sweden were made available by SNIC. G.B. acknowledges financial support by the Swedish Research Council (grant number 621-2013-5784).

\section{REFERENCES}

Augier, P., Billant, P. \& Chomaz, J.-M. 2015 Stratified turbulence forced with columnar dipoles: numerical study. J. Fluid Mech. 769, 403-443.

Barry, M.E., Ivey, G.N., Winters, K.B. \& Imberger, J. 2001 Measurements of diapycnal diffusivities in stratified fluids. J. Fluid Mech. 442, 267-291.

Bartello, P. \& Tobias, S.M. 2013 Sensitivity of stratified turbulence to the buoyancy Reynolds number. J. Fluid Mech. 725, 1-22.

Billant, P. \& Chomaz, J.-M. 2001 Self-similarity of strongly stratified inviscid flows. Phys. Fluids 13 (6), 1645-1651.

Brethouwer, G., Billant, P., Lindborg, E. \& Chomaz, J.-M. 2007 Scaling analysis and simulation of strongly stratified turbulent flows. J. Fluid Mech. 585, 343-368.

DE BRUYN Kops, S.M. 2015 Classical scaling and intermittency in strongly stratified Boussinesq turbulence. J. Fluid Mech. 775, 436-463.

Davis, K.A. \& Moninsmith, S.G 2011 The modification of bottom boundary layer turbulence and mixing by internal waves shoaling on a barrier reef. J. Phys. Oceanog. 41, 2223-2241.

Donzis, D.A., Sreenivasan, K.R. \& Yeung, P.K. 2005 Scalar dissipation rate and dissipative anomaly in isotropic turbulence. J. Fluid Mech. 532, 199-216.

Holford, J.M. \& Linden, P.F. 1999 Turbulent mixing in a stratified fluid. Dyn. Atmos. Oceans 30, 173-198.

Ivey, G.N., Winters, K.B. \& Koseff, J.R. 2008 Density stratification, turbulence, but how much mixing? Annu. Rev. Fluid Mech. 40, 169-184.

Kato, H. \& Philipps, O.M. 1969 On the penetration of a turbulent layer into stratified fluid. J. Fluid Mech. 37 (4), 643-655.

LindBorG, E. 2006 The energy cascade in a strongly stratified fluid. J. Fluid Mech. 550, 207242.

Lindborg, E. \& Brethouwer, G. 2008 Vertical dispersion by stratified turbulence. J. Fluid Mech. 614, 303-314.

Lozovatsky, I.D. \& Fernando, H.J.S. 2013 Mixing efficiency in natural flows. Phyl. Trans. R. Soc. A 371 (20120213).

Maffioli, A. \& Davidson, P.A. 2015 Dynamics of stratified turbulence decaying from a high buoyancy Reynolds number. J. Fluid Mech. 786, 210-233.

Mater, B.D. \& Venayagamoorthy, S.K. 2014 The quest for an unambiguous parameterization of mixing efficiency in stably stratified geophysical flows. Geophys. Res. Letters 41, 4646-4653. 
Monti, P., Fernando, H.J.S., Princevac, M., Chan, W.C., Kowalewski, T.A. \& PARDYJAK, E.R. 2002 Observations of flow and turbulence in the nocturnal boundary layer over a slope. J. Atmos. Sci. 59 (17), 2513-2534.

Oglethorpe, R.L.F., Caulfield, C.P. \& Woods, A.W. 2013 Spontaneous layering in stratified turbulent Taylor-Couette flow. J. Fluid Mech. 721, R3.

Olsthoorn, J. \& Dalziel, S.B. 2015 Vortex-ring-induced stratified mixing. J. Fluid Mech. 781, $113-126$.

Osborn, T.R. 1980 Estimates of the local rates of vertical diffusion from dissipation measurements. J. Phys. Oceanog. 10, 83-89.

Osborn, T.R. \& Cox, C.S. 1972 Oceanic fine structure. Geophys. Fluid Dyn. 3, 321-345.

Park, J.-G., Whitehead, J.A. \& Gnanadeskian, A. 1994 Turbulent mixing in stratified fluids: layer formation and energetics. J. Fluid Mech. 279, 279-311.

Riley, J.J. \& De Bruyn Kops, S.M. 2003 Dynamics of turbulence strongly influenced by buoyancy. Phys. Fluids 15 (7), 2047-2059.

Riley, J.J. \& LELONG, M.-P. 2000 Fluid motions in the presence of strong stable stratification. Annu. Rev. Fluid Mech. 32, 613-657.

Rogallo, R.S. 1981 Numerical experiments in homogeneous turbulence. NASA Technical Memorandum 81315. NASA Ames Research Center.

Salehipour, H. \& Peltier, W.R. 2015 Diapycnal diffusivity, turbulent Prandtl number and mixing efficiency in Boussinesq stratified turbulence. J. Fluid Mech. 775, 464-500.

Shin, L.H., Koseff, J.R., Ivey, G.N. \& Ferziger, J.H. 2005 Parameterization of turbulent fluxes and scales using homogeneous sheared stably stratified turbulence simulations. $J$. Fluid Mech. 525, 193-214.

Sмyтн, W.D. \& Moum, J.N. 2000 Length scales of turbulence in stably stratified mixing layers. Phys. Fluids 12 (6), 1327-1342.

Smyth, W.D., NAsh, J.D., \& Moum, J.N. 2005 Differential diffusion in breaking KelvinHelmholtz Billows. J. Phys. Ocean. 35, 1004-1022.

TAYlor, G.I. 1935 Statistical theory of turbulence. Proc. R. Soc. A 151, 421-478.

TURner, J.S. 1968 The influence of molecular diffusivity on turbulent entrainment across a density interface. J. Fluid Mech. 33 (4), 639-656.

Turner, J.S. 1973 Buoyancy effects in fluids. Cambridge University Press.

Waite, M.L. 2011 Stratified turbulence at the buoyancy scale. Phys. Fluids 23 (6).

Waite, M.L. \& Bartello, P. 2004 Stratified turbulence dominated by vortical motion. J. Fluid Mech. 517, 281-308.

Walter, R.K., SquibB, M.E., Woodson, C.B., Koseff, J.R. \& Monismith, S.G. 2014 Stratified turbulence in the nearshore coastal ocean: dynamics and evolution in the presence of internal bores. J. Geophys. Res. Oceans 119, 8709-8730.

Wells, M., Cenedese, C. \& Caulfield, C.P. 2010 The relationship between flux coefficient and entrainment ratio in density currents. J. Phys. Oceanog. 40, 2713-2727. 